\title{
McDonald versus Shirodkar cerclage technique in the prevention of preterm birth. A systematic review and meta-analysis
}

\author{
Liam McAuliffe $^{1}$, Ashad Issah ${ }^{1}$, Rosanna Diacci ${ }^{1}$, Kimberley Williams ${ }^{1}$, Anne-Marie \\ Aubin ${ }^{1}$, Jason Phung ${ }^{1}$, Carol Wang ${ }^{1}$, Alexander Maouris ${ }^{2}$, Sebastian Leathersich ${ }^{3}$, \\ Panayiotis Maouris ${ }^{3}$, and Craig Pennell ${ }^{1}$ \\ ${ }^{1}$ The University of Newcastle \\ ${ }^{2}$ Sir Charles Gairdner Hospital \\ ${ }^{3}$ King Edward Memorial Hospital
}

February 21, 2021

\begin{abstract}
Background: Cervical cerclage has been used for decades to decrease rates of preterm birth. The Shirodkar and McDonald cerclage are the two most commonly used cerclage techniques with no current consensus on the preferred technique. Objective: To compare the efficacy of the two techniques. Search strategy: Studies were sourced from six electronic databases and reference lists. Selection criteria: Studies including women with a singleton pregnancy, requiring a cervical cerclage, using either the Shirodkar or McDonald technique that ran comparative analyses between the two techniques. Data collection and analysis: The primary outcome was preterm birth before 37 weeks, with sub analyses at 28, 32, 34 and 35 weeks. Secondary data was also collected on neonatal, maternal and obstetric outcomes. Main results: Seventeen papers were included - analysis showed the Shirodkar group had significantly less chance of preterm birth before 37 weeks (RR 0.91, 95\% CI 0.85-0.98). This finding is reinforced by statistically significant reduction in rates of preterm birth before 37, 35, 34 and 32 weeks, PPROM (RR 0.87 , $95 \%$ CI 0.77 - 0.99), difference in cervical length (mean difference 5.25, 95\% CI 4.68-5.83), cerclage to delivery interval (mean difference 10.79, 95\% CI 8.20-13.38), and an increase in birthweight (mean difference 348 grams, 95\% CI 291-406) in the Shirodkar group. Conclusion: Shirodkar cerclage leads to a significant reduction in preterm birth and delivers better maternal and neonatal outcomes when compared to McDonald cerclage. Funding: No funding was required for this review. Keywords: Cervical, Stitch, Cerclage, McDonald, Shirodkar, Preterm birth
\end{abstract}

McDonald versus Shirodkar cerclage technique in the prevention of preterm birth. A systematic review and meta-analysis. Authors Liam McAuliffe (Liam.McAuliffe@uon.edu.au) ${ }^{1}$ Ashad Issah (Ashad.Issah@uon.edu.au) ${ }^{1}$ Rosanna Diacci (Rosanna.Diacci@uon.edu.au) ${ }^{1}$ Kimberley P Williams (Kimberley.P.Williams@uon.edu.au) ${ }^{1}$ Anne-Marie Aubin (Anne-Marie.Aubin@uon.edu.au) ${ }^{1}$ Jason Phung (Jason.Phung@health.nsw.gov.au) ${ }^{1}{ }^{2}$, ${ }^{3}$ Carol Wang (Carol.Wang@newcastle.edu.au) ${ }^{1,2}$ Alexander Maouris (Alexander.maouris@health.wa.gov.au) ${ }^{4}$ Sebastian Leathersich (Sebastian.leathersich@health.wa.gov.au) ${ }^{5}$ Panos Maouris (Panayiotis.Maouris@health.wa.gov.au) ${ }^{5} \mathrm{Craig}$ E Pennell (Craig.Pennell@newcastle.edu.au) ${ }^{1,2,3}{ }^{1}$ School of Medicine and Public Health, The University of Newcastle, Callaghan, New South Wales, Australia. ${ }^{2}$ Mothers and Babies Research Centre, Hunter Medical Research Institute, New Lambton Heights, New South Wales, Australia. ${ }^{3}$ Maternity and Gynaecology John Hunter Hospital, New Lambton Heights, New South Wales, Australia. ${ }^{4}$ Sir Charles Gairdner Hospital, Perth, Western Australia, Australia. ${ }^{5}$ King Edward Memorial Hospital, Subiaco, Western Australia, Australia.

Corresponding author: Craig Pennell 
Address: Lot 1 Kookaburra Circuit, New Lambton Heights, NSW, Australia.

Phone: (02) 40420546

Shortened running title: McDonald versus Shirodkar cerclage technique

Word count: 2939

\title{
Abstract
}

Background: Cervical cerclage has been used for decades to decrease rates of preterm birth. The Shirodkar and McDonald cerclage are the two most commonly used cerclage techniques with no current consensus on the preferred technique. Objective: To compare the efficacy of the two techniques. Search strategy: Studies were sourced from six electronic databases and reference lists. Selection criteria: Studies including women with a singleton pregnancy, requiring a cervical cerclage, using either the Shirodkar or McDonald technique that ran comparative analyses between the two techniques. Data collection and analysis: The primary outcome was preterm birth before 37 weeks, with sub analyses at 28, 32, 34 and 35 weeks. Secondary data was also collected on neonatal, maternal and obstetric outcomes.Main results: Seventeen papers were included - analysis showed the Shirodkar group had significantly less chance of preterm birth before 37 weeks (RR 0.91, 95\% CI 0.85-0.98). This finding is reinforced by statistically significant reduction in rates of preterm birth before 37, 35, 34 and 32 weeks, PPROM (RR 0.87, 95\% CI $0.77-0.99$ ), difference in cervical length (mean difference 5.25, 95\% CI 4.68-5.83), cerclage to delivery interval (mean difference 10.79, 95\% CI 8.20-13.38), and an increase in birthweight (mean difference 348 grams, 95\% CI 291-406) in the Shirodkar group. Conclusion:Shirodkar cerclage leads to a significant reduction in preterm birth and delivers better maternal and neonatal outcomes when compared to McDonald cerclage. Funding: No funding was required for this review.Keywords: Cervical, Stitch, Cerclage, McDonald, Shirodkar, Preterm birth

\section{Tweetable Abstract}

'Shirodkar cerclage provides better outcomes than McDonald in the prevention of preterm birth.'

\section{Introduction}

Preterm birth (PTB) is responsible for an estimated one million neonatal deaths per year, making it the leading cause of mortality in children under five years(1). Despite increasing international research, PTB remains an unresolved obstetric complication of pregnancy, affecting $5-13 \%$ of pregnant women(2). Children born preterm are at a higher risk of respiratory distress syndrome (RDS), intraventricular haemorrhage, necrotising enterocolitis and retinopathy of prematurity(3). Preterm Birth is also associated with increased maternal morbidity with higher rates of obstetric haemorrhage, infection and intensive care unit admission, likely related to increased operative delivery(4).

A short cervical length $(<25 \mathrm{~mm})$ is a good predictor of PTB, with a $31.2 \%$ to $41.3 \%$ risk of PTB, if present between 18 and 24 weeks $(5,6)$. In these women, management options include vaginal progesterone $(7)$ or cervical cerclage $(5,8)$. Cervical cerclage has been used since the 1950's and two methods are in common use. The McDonald technique is a simpler purse-string suture around the cervix whereas the Shirodkar technique involves colpotomy and bladder dissection with the aim of a higher suture placement.

There is currently a lack of consensus on the superiority of the McDonald or Shirodkar technique of cervical cerclages and there are no current guidelines or agreement on which technique is recommended. In the absence of emerging therapies to prevent PTB, combined with the increasing rate of PTB worldwide and in Australia, there is a great need to maximise the effect of the currently available treatments. This review aims to determine if one technique provides a greater reduction in preterm birth rates.

\begin{abstract}
Aim
To synthesise existing quantitative evidence comparing McDonald with Shirodkar cervical cerclage techniques to determine which correlates to better maternal and neonatal outcomes. This systematic review will answer
\end{abstract}


the following question regarding women requiring prophylactic cervical cerclage in singleton pregnancy: Is there a difference between the McDonald and the Shirodkar cerclage techniques in the prevention of PTB and other significant maternal or neonatal outcomes?

\section{Methods}

A protocol paper detailing the methods for this systemic review and meta-analysis have been previously published(9). Our systematic review protocol was submitted to the International Prospective Register of Systematic Reviews (PROSPERO) on the 20th of April 2020 and accepted on the $6^{\text {th }}$ July 2020 (registration number CRD42020177386). We have presented the data using the Preferred Reporting Items for Systematic Reviews and Meta-Analyses (PRISMA) statement(10), the Meta-Analyses of Observational Studies checklist (MOOSE)(11) and the Cochrane Handbook for Systematic Reviews of Interventions(12). Information regarding registration can be accessed from http://www.crd.york.ac.uk/PROSPERO.

\section{Eligibility Criteria:}

Eligibility of studies included in this systematic review was based on pre-planned inclusion and exclusion criteria applied to each of the following domains: participant, exposure, comparator, study type and outcome.

\section{Participants:}

The review considered all studies that included pregnant women undergoing McDonald or Shirodkar cervical cerclage for prevention of PTB. Studies were excluded if they included women with multiple gestation pregnancies.

\section{Intervention :}

Studies which compared the McDonald and Shirodkar techniques of cervical cerclage as a prophylactic procedure.

\section{McDonald Cerclage}

In the McDonald approach a suture is placed around the cervix in purse-string fashion and securely tied anteriorly. The McDonald approach requires no dissection into para-cervical tissues(13, 14).

\section{Shirodkar Cerclage}

The Shirodkar technique involves a transverse anterior colpotomy, dissection of the bladder up to the internal cervical os and a posterior colpotomy with dissection of areola and peritoneum upwards to the internal os. The suture is placed subcutaneously and the knot tied in the posterior defect and buried under the vaginal epithelium $(13,15,16)$. Later modifications do not require a posterior colpotomy and place the knot exterior to the vaginal mucosal for ease of removal(17). Whilst technically more challenging, the rationale of this technique is to allow more proximal placement of cerclage closer to the internal os. A number of other modifications have been reported which simplify the Shirodkar technique by utilising a clamp on the paracervical tissues for more accurate suture needle placement(18) or avoiding a posterior colpotomy and suture burial(17). For the purpose of this study both the original technique described by Shirodkar and the modified techniques were included.

\section{Types of studies:}

This review accepted randomised control trials, pseudo-randomised control trials, non-randomised experimental control trials and cohort studies. All papers included had to compare the co-interventions, McDonald and Shirodkar cerclage.

Search Strategy: 
Six electronic bibliographic databases were searched for eligible, peer-reviewed literature: Medline (Ovid), EMBASE (Ovid), PsycINFO (Ovid), Scopus, CINAHL (EBSCOhost), and Cochrane Library (Wiley). Reference lists of included studies were screened and references in academic textbooks were also reviewed. Where studies were unable to be sourced contact was attempted with the corresponding author. A more detailed database search strategy is described in Appendix S1.

Data collection and analysis

\section{Study Selection}

The titles and abstracts were reviewed using Endnote(19) and Covidence(20). Studies that did not meet the criteria based on abstracts were excluded (authors A-M.A and L.M.) and full texts of remaining articles were sourced and screened (A-M.A and R.D.). No language restriction was set, all non-English included studies were translated. Included studies were critically appraised (by L.M and A.I.) and data extracted using a standardised electronic form (by R.D. and K.P.W.). At all levels of screening, any discrepancies were moderated by a third senior reviewer (C.E.P.).

Assessment of risk of bias

To facilitate the assessment of possible risk of bias for each study, two independent reviewers (A.I and L.M) assessed each paper using the Cochrane Collaboration tool for assessing the risk of bias (ROBINS-I \& RoB $2)(21,22)$ for non-randomised and randomised studies respectively(12).

Cochrane GRADE Assessment

Quality of evidence for our primary outcome was judged using the GRADE tool by two independent reviewers (A-M.A. and K.P.W.)(23).

\section{Outcomes}

Primary outcome

The primary outcome was PTB less than 37 weeks gestation.

Secondary outcomes

Secondary outcomes included PTB less than 28, 32, 34, and 35 weeks. Maternal secondary outcomes examined were rates of preterm premature rupture of the membranes

(PPROM), chorioamnionitis (clinical or laboratory diagnosis), cervical laceration and stenosis, caesarean section delivery (excluding planned or elective), number of days between cerclage and delivery, intraoperative membrane rupture at time of cerclage, cases requiring repeat cerclage and difference in cervical length before and after cerclage. Neonatal outcomes included birthweight, Apgar score $<7$, and neonatal mortality, which included mortality up to one month post-delivery or neonatal survival (this figure was then inverted to mortality). Thorough definitions of these outcomes are referenced in the protocol paper(9).

\section{Data synthesis}

A meta-analysis was performed by pooling studies together using RevMan(24) and Covidence software(20). The heterogeneity of data was examined using forest plots and quantified throughout calculation of the $\mathrm{I}^{2}$ value. An $\mathrm{I}^{2}$ of greater than or equal to $50 \%$ was used to indicate substantial heterogeneity and a randomeffects model was used. For all $\mathrm{I}^{2}$ less than $50 \%$, a fixed effects model was used. Outcomes with less than five studies were analysed using a fixed effects model(25). For reporting consistency between outcomes, we made the McDonald intervention the reference set for all analyses, standardising the direction of effect across all primary and secondary outcomes.

Measures of treatment effect 
Where applicable, trial data were combined and reported using meta-analyses using the standard estimation of: 1) risk ratio (RR) and $95 \%$ confidence intervals (CI) for dichotomous outcome variables, and 2) mean differences (MDs) or standardised mean differences (SMDs) and 95\% CIs for continuous outcome variables.

Sensitivity Analysis

Sensitivity analysis was conducted on the primary outcomes - PTB less than 37 weeks gestation. This was performed by removing studies with an overall high risk of bias to examine their impact on the effect estimate.

\section{Results}

\section{Study selection and characteristics}

Searches of databases identified a total of 178 studies and manual searches identified one additional publication (Figure 1). Seventy-five papers were removed due to duplication, 46 were excluded on title and abstract review, and an additional 41 were excluded at the level of full text review. Of those excluded at full text screening, 14 were the wrong study design, and two were unable to be sourced. Of the remaining 23: a total of eight did not compare Shirodkar and McDonald, a further 15 did not report usable outcomes that were comparable to the outcomes of interest in this review, and another two reported on the wrong population. This process left a total of 17 studies to be included in the meta-analysis (Figure 1).

A summary of the of key characteristics of the included studies is presented in Table 1. In brief, 17 included studies which consisted of a total of 2063 participants were included in this review; of these, 16 (94.1\%) of them were retrospective cohort studies, while one (5.9\%) was a multicentre, retrospective study which used data from four randomised control trials. Of the retrospective cohort studies, data were retrieved from women's records and hospital data collected between 1957 and 2014. The sample sizes for the included studies ranged from 25 to 374 and all studies reported quantitative data.

\section{Risk of bias and quality of evidence}

Table $2 \mathrm{~A}$ and $2 \mathrm{~B}$ show the level of risk in each of the domains of bias.

Risk of bias for individual studies was determined using the Cochrane ROBINS-I for cohort studies and RoB-2 for the randomised control trials $(21,22)$.

In examining bias risk, it was found that more dated trials tended to have a higher risk of bias whereas more recent ones appeared to show more favourable risk of bias. This is likely due to improved trial design and reporting. Thirteen papers (76.5\%) were judged to be at serious risk of bias, six (35.3\%) at moderate risk, and two (11.7\%) at low risk. The quality of evidence for the primary outcome, birth before 37 weeks, was assessed using GRADE and was ranked as low quality.

\section{Birth before 37 weeks}

The association between technique of cervical cerclage and the primary outcome of birth less than 37 weeks is shown in Figure 2. Twelve studies (70.5\%) reported data on this outcome. There were significantly lower rates of birth before 37 weeks in the Shirodkar group when compared to the McDonald group (RR 0.91, 95\% CI 0.85-0.98).

Removing studies with serious risk of bias improved the quality of evidence from low to moderate and did not alter the results. No subgroup analysis was undertaken.

Birth $<35,<34,<32,<28$, number of days between cerclage and birth

Consistent with the primary outcome, rates of preterm birth before 35, 34 and 32 weeks all were significantly lower in the Shirodkar group. Preterm birth before 28 weeks was the only one of these outcomes to show no difference between the cerclage techniques. 
The Shirodkar technique also associated with significantly longer interval between cerclage and birth (mean 10.79 days, CI 8.20-13.38, Table 3 ).

\section{Neonatal outcomes (neonatal mortality and birthweight)}

There was no difference between the two techniques in neonatal mortality (RR 1.01, 95\% CI 0.97 - 1.04). The birthweight in the Shirodkar group was significantly greater than the McDonald group (mean difference 348 grams, 95\% CI 291-405, Table 3).

\section{Maternal outcomes (PPROM, chorioamnionitis, cervical laceration, caesarean section and cer- vical length)}

The rate of PPROM was found to be significantly lower in the Shirodkar group when compared to the McDonald group (RR 0.87, 95\% CI 0.77 - 0.99). The Shirodkar technique group also showed a significantly greater increase in the post cerclage cervical length (mean difference 5.25, 95\% CI 4.68-5.83).

There was no difference between the two techniques in the rates of chorioamnionitis (RR $0.96,95 \%$ CI 0.90 - 1.02), cervical laceration (RR 1.04, 95\% CI $0.98-1.10$ ), or caesarean section (RR 1.07, 95\% CI $0.94-$ 1.22 , Table 3 ).

\section{Discussion}

\section{Main findings}

Cervical cerclage is an effective method of PTB prevention(26, 27). Until now, however, there has not been sufficient evidence from individual small studies to reach a consensus opinion amongst experts that one cerclage technique is more effective than the other. This systematic review and meta-analysis of all available data shows that pregnancies requiring a cervical cerclage are significantly less likely to result in preterm birth when the Shirodkar technique of cerclage is utilised compared to the McDonald approach. The number needed to treat (NNT) was calculated using the risk difference. It was estimated that 38 (95\% CI 23-171) additional Shirodkar cerclages would be required in order to prevent one additional preterm birth $<37$ weeks, while an additional 28 (95\% CI 18-56) Shirodkar cerclages would need to be performed to prevent one birth $<32$ weeks $(28,29)$. This finding is reinforced by a statistically significant reduction in rates of preterm birth before 37, 35, 34 and 32 weeks, PPROM, difference in cervical length, cerclage to delivery interval and an increase in birthweight in the Shirodkar group. Secondary analyses showed the greatest statistical differences in PTB exist at $<32$ and $<34$ weeks with approximately three-fold reductions in PTB rates when a Shirodkar cerclage was used.

It is well established that the risk of spontaneous PTB is increased for women with a short cervix on transvaginal ultrasound(30-32). Previous studies have shown a correlation between increased cervical length post cerclage and later gestation at delivery $(33,34)$. A cerclage height of at least $18 \mathrm{~mm}$ (measured from the cerclage to the external os in a mid sagittal plane on transvaginal ultrasound) has been shown to be associated with a reduction in PTB when compared to cerclages placed closer to the external os(35). In two separate publications by Sheib et al. and Miroshnichenko et al., the McDonald cerclage has been shown to fail to achieve this height in the majority of women $(35,36)$. The Shirodkar approach places the cerclage higher and closer to the internal os, hence it is more likely to result in a longer post-cerclage cervical length. This is supported by the two included studies comparing cervical length post cerclage $(37,38)$ in this review, which showed a significant increase in cervical length post cerclage for the Shirodkar technique when compared to the McDonald approach (mean difference $5.25 \mathrm{~mm}, 95 \%$ CI: 4.68-5.83). Intuitively, the ability to achieve a longer cervix with cerclage placement closest to the internal os is more likely with the Shirodkar procedure as it allows clinical estimation of the internal os after dissection of the urinary bladder away from the cervix.

Respiratory distress syndrome (RDS) was not included in the final analysis as only one included paper reported on the outcome. However, the study by Wong et al., showed a statistically significant reduction in RDS rates when the Shirodkar technique was used. This is in keeping with literature which links increasing prematurity with RDS rates. 
The interval between cerclage and birth, was significantly longer in the Shirodkar cerclage. This outcome was reported on by two studies; one of which favoured McDonald(39) and the other favouring Shirodkar(40). It should, however, be noted that in the study by Odibo et al. that favoured the McDonald cerclage, the McDonald group had a significantly longer cervical length at entry into the study and less advanced gestational age when compared to the Shirodkar group (20 versus 23 weeks)(39).

Even though the Shirodkar technique requires greater surgical expertise than the McDonald approach, the procedure complication rate is generally low $(26)$. This review was not able to compare rates of intraoperative rupture of membranes or repeat cerclage due to small numbers. However, there was no difference in the rates of cervical laceration between the two techniques. Furthermore, PPROM, arguably an important delayed technique-related complication, occurs less frequently with the Shirodkar technique without a previously reported increase in the Caesarean section rate(37, 40-43).

\section{Strengths and Limitations}

One of the key strengths of this meta-analysis is the uniformity of the results supporting the Shirodkar technique.

A significant limitation of this meta-analysis is the high risk of bias of many of the included studies. The overall quality of the evidence for the primary outcome was low; however, sensitivity analysis removing the impact of papers with high risk of bias improved the quality to moderate without altering the results for the primary outcome. There were two included papers judged to be of low risk of bias and both reported lower rates of PTB in the Shirodkar groups(40,43). More than half the included studies were published prior to 2000, with 13 studies classified as having a high risk of bias; however, sensitivity analyses did not alter our primary results. All papers included were cohort studies except for one which used data from four randomised control trials(44), which reported lower rates of PTB in the Shirodkar arm when compared to both McDonald cerclage and bed rest.

As a result of the sparse number of events and unreported outcomes in the studies examined in this review, we were not able to report on some outcomes mentioned in our protocol paper(9). These included Apgar score, intra-operative membrane rupture, cervical stenosis, repeat cerclage rate, RDS rate and a sub-analysis of preterm birth at $<36$ weeks. It should also be noted that while cervical length and neonatal respiratory distress syndrome were included, the sample size in these analyses was small (Table 3) and these results should be interpreted with caution.

Another limitation was that all but one included paper did not control for the effect of surgical experience of different operators. The only study with a single surgeon for all procedures, who was equally experienced in both techniques, reported statistically significant better outcomes for PTB with the Shirodkar approach(43).

This review reported results, favouring the Shirodkar approach over the McDonald approach, which were consistent in effect sizes and direction of effects. The overall quality of the studies in the review, however, was low with the risk of bias rated from between moderate to high. Thus, there is a need for further well designed, randomised control studies to compare the outcomes between different cervical cerclage techniques.

\section{Interpretation}

Current guidelines from the Royal College of Obstetricians and Gynaecologists and the Society of Obstetricians of Gynaecologists of Canada(45), advise that surgical technique should depend on the experience of, and be at the discretion of, the surgeon(46). The data presented in this study suggest that a wellconstructed randomised control trial comparing surgical techniques is required. Until then, both surgical techniques should continue to be taught to training obstetricians. Individual circumstances will vary for each woman and the final decision should be left to the discretion of the obstetrician.

\section{Conclusion}

The Shirodkar cerclage technique achieves greater cerclage height and longer interval between time at cerclage insertion to delivery, leading to increased birthweight and a reduction in PPROM and PTB when compared 
to the McDonald approach. By choosing the Shirodkar technique rather than the McDonald technique, one additional preterm birth would be prevented for every 38 cerclage procedures. Clinicians should consider these results when deciding which cerclage technique to utilise; however, further unbiased high-quality studies are needed to provide stronger supporting evidence that the Shirodkar approach has the potential to achieve better outcomes when compared to the McDonald approach. We recommend that obstetricians in training should be taught both surgical approaches where possible and decisions about which technique to use should be individualised to the woman.

\section{Acknowledgements}

The authors would like to thank Mr Jack McAuliffe and Dr Daniel Barker for their statistical advice and Ms Joanne Davies for her research advice.

\section{Disclosure of Interests}

J.P. is supported by the Hunter New England Health Local Health District Clinical and Health Service Research Fellowship Scheme. The authors declare they have no other competing interests.

\section{Contribution to authorship}

L.M., A.I., K.P.W., R.D., A-M.A., J.P. and C.E.P. led the development of the manuscript. A.M., S.L., P.M., J.P. and C.E.P. designed the concept of the review and drafted the manuscript. C.W. provided statistical analysis advice and drafting. J.P., P.M. and C.E.P. advised on obstetric care.

\section{Funding}

No funding was sourced for the writing or publication of this manuscript.

\section{References}

1. Liu L, Oza S, Hogan D, Chu Y, Perin J, Zhu J, et al. Global, regional, and national causes of under-5 mortality in 2000-15: an updated systematic analysis with implications for the Sustainable Development Goals. The Lancet. 2016;388(10063):3027-35.

2. Blencowe H, Cousens S, Oestergaard MZ, Chou D, Moller A-B, Narwal R, et al. National, regional, and worldwide estimates of preterm birth rates in the year 2010 with time trends since 1990 for selected countries: a systematic analysis and implications. The Lancet. 2012;379(9832):2162-72.

3. Ward RM, Beachy JC. Neonatal complications following preterm birth. British Journal of Obstetrics and Gynaecology. 2003;110:8-16.

4. Reddy UM, Rice MM, Grobman WA, Bailit JL, Wapner RJ, Varner MW, et al. Serious maternal complications after early preterm delivery (24-33 weeks' gestation). American Journal of Obstetrics and Gynecology. 2015;213(4):538. e1- e9.

5. Berghella V, Rafael TJ, Szychowski JM, Rust OA, Owen J. Cerclage for short cervix on ultrasonography in women with singleton gestations and previous preterm birth: a meta-analysis. Obstetrics and Gynecology. 2011;117(3):663-71.

6. Souza RT, Costa ML, Mayrink J, Feitosa FE, Rocha Filho EA, Leite DF, et al. Clinical and epidemiological factors associated with spontaneous preterm birth: a multicentre cohort of low risk nulliparous women. Scientific Reports. 2020;10(1):1-10.

7. Romero R, Conde-Agudelo A, Da Fonseca E, O'Brien JM, Cetingoz E, Creasy GW, et al. Vaginal progesterone for preventing preterm birth and adverse perinatal outcomes in singleton gestations with a short cervix: a meta-analysis of individual patient data. American Journal of Obstetrics and Gynecology. 2018;218(2):161-80.

8. MRC/RCOG Working Party on Cervical Cerclage, Macnaughton M, Chalmers I, Dubowitz V, Dunn P, Grant A, et al. Final report of the Medical Research Council/Royal College of Obstetricians and Gynae- 
cologists multicentre randomised trial of cervical cerclage. British Journal of Obstetrics and Gynaecology. 1993;100(6):516-23.

9. Issah A, Diacci R, Williams KP, Aubin A, McAuliffe L, Phung J, et al. McDonald versus Shirodkar Cerclage technique in women requiring a prophylactic cerclage: a systematic review and meta-analysis protocol. BMC Systematic Reviews. (Under Review).

10. Moher D, Shamseer L, Clarke M, Ghersi D, Liberati A, Petticrew M, et al. Preferred reporting items for systematic review and meta-analysis protocols (PRISMA-P) 2015 statement. Systematic Reviews. $2015 ; 4(1): 1$.

11. Stroup DF, Berlin JA, Morton SC, Olkin I, Williamson GD, Rennie D, et al. Meta-analysis of observational studies in epidemiology: a proposal for reporting. JAMA. 2000;283(15):2008-12.

12. Higgins J, Thomas J. Cochrane Handbook for Systematic Reviews of Interventions version 6.0 (updated July 2019). Chandler J CM, Li T, Page MJ, Welch VA editor 2019.

13. Jones H RJ. Te Lindes Operative Gynecology: Lippincott, Williams \& Wilkins; 2015.

14. McDonald I. Suture of the cervix for inevitable miscarriage. British Journal of Obstetrics and Gynaecology. 1957;64(3):346-50.

15. Barter RH, Dusbabek JA, Tyndal CM, Erkenbeck RV. Further experiences with the Shirodkar operation. American Journal of Obstetrics and Gynecology. 1963;85(6):792-805.

16. Shirodkar V. A new method of operative treatment for habitual abortions in the second trimester of pregnancy. Antiseptic. 1955;52:299-300.

17. Caspi E, Schneider DF, Mor Z, Langer R, Weinraub Z, Bukovsky I. Cervical internal os cerclage: description of a new technique and comparison with Shirodkar operation. American Journal of Perinatology. 1990;7(04):347-9.

18. Druzin M, Berkeley A. A simplified approach to Shirodkar cerclage procedure. Surgery, Gynecology \& Obstetrics. 1986;162(4):375-6.

19. Endnote X9. Clarivate Analytics. Philadelphia, United States: Thomson Reuters; 2019.

20. Covidence systematic review software Melbourne, Australia: Veritas Health Innovation; [

21. Sterne JA, Hernan MA, Reeves BC, Savović J, Berkman ND, Viswanathan M, et al. ROBINS-I: a tool for assessing risk of bias in non-randomised studies of interventions. BMJ. 2016;355:i4919.

22. Sterne JA, Savović J, Page MJ, Elbers RG, Blencowe NS, Boutron I, et al. RoB 2: a revised tool for assessing risk of bias in randomised trials. BMJ. 2019;366.

23. Schünemann H BJ, Guyatt G, Oxman A, editor(s). Handbook for grading the quality of evidence and the strength of recommendations using the GRADE approach (updated October 2013) GRADE Working Group, 2013. [Available from: guidelinedevelopment.org/app/handbook/handbook.html].

24. Review Manager (RevMan). [Computer program]. Copenhagen The Nordic Cochrane Centre: The Cochrane Collaboration; 2014.

25. Higgins J, Green S (editor). Incorporating heterogeneity into random-effects models Cochrane Handbook for Systematic Reviews of Interventions Version 5.1.0 The Cochrane Collaboration; 2011.

26. Alfirevic Z, Stampalija T, Medley N. Cervical stitch (cerclage) for preventing preterm birth in singleton pregnancy. Cochrane Database of Systematic Reviews. 2017(6).

27. National Institute of Health and Care Excellence. Preterm labour and birth (NICE guideline 25) 2015 (updated 2019). 
28. Mendes D, Alves C, Batel-Marques F. Number needed to treat (NNT) in clinical literature: an appraisal. BMC Medicine. 2017;15(1):112.

29. Higgins J, Green S (editor). Computing NNT from a risk difference (RD). Cochrane Handbook for Systematic Reviews of Interventions Version 5.1.0 London: The Cochrane Collaboration; 2011.

30. Iams JD, Goldenberg RL, Meis PJ, Mercer BM, Moawad A, Das A, et al. The Length of the Cervix and the Risk of Spontaneous Premature Delivery. New England Journal of Medicine. 1996;334(9):567-73.

31. To M, Skentou C, Royston P, Yu C, Nicolaides K. Prediction of patient-specific risk of early preterm delivery using maternal history and sonographic measurement of cervical length: a population-based prospective study. Ultrasound in Obstetrics and Gynecology. 2006;27(4):362-7.

32. To MS, Fonseca EB, Molina FS, Cacho AM, Nicolaides KH. Maternal characteristics and cervical length in the prediction of spontaneous early preterm delivery in twins. American Journal of Obstetrics and Gynecology. 2006;194(5):1360-5.

33. Dijkstra K, Funai EF, O'Neill L, Rebarber A, Paidas MJ, Young BK. Change in cervical length after cerclage as a predictor of preterm delivery. Obstetrics and Gynecology. 2000;96(3):346-50.

34. Sim S, Da Silva Costa F, Araujo Junior E, Sheehan PM. Factors associated with spontaneous preterm birth risk assessed by transvaginal ultrasound following cervical cerclage. Australian and New Zealand Journal of Obstetrics and Gynaecology. 2015;55(4):344-9.

35. Scheib S, Visintine JF, Miroshnichenko G, Harvey C, Rychlak K, Berghella V. Is cerclage height associated with the incidence of preterm birth in women with an ultrasound-indicated cerclage? American Journal of Obstetrics and Gynecology. 2009;200(5):e12-e5.

36. Miroshnichenko G, Visintine JF, Suhag A, Gerson A, Berghella V. Is cerclage height associated with the incidence of preterm birth in women with a history-indicated cerclage? American Journal of Perinatology. 2011;28(01):083-6.

37. Perrotin F, Marret H, Ayeva-Derman M, Alonso A, Lansac J, Body G. Second trimester cerclage of short cervixes: which technique to use? A retrospective study of 25 cases. Journal de Gynecologie, Obstetrique et Biologie de la Reproduction. 2002;31(7):640-8.

38. Rozenberg P, Senat M-V, Gillet A, Ville Y. Comparison of two methods of cervical cerclage by ultrasound cervical measurement. The Journal of Maternal-Fetal and Neonatal Medicine. 2003;13(5):314-7.

39. Odibo AO, Berghella V, To MS, Rust OA, Althuisius SM, Nicolaides KH. Shirodkar versus McDonald cerclage for the prevention of preterm birth in women with short cervical length. American Journal of Perinatology. 2007;24(01):055-60.

40. Figueroa R, Crowell R, Martinez A, Morgan M, Wakefield D. McDonald versus Shirodkar cervical cerclage for the prevention of preterm birth: impact of body mass index. The Journal of Maternal-Fetal and Neonatal Medicine. 2019;32(20):3408-14.

41. Bartolo S, Garabedian C, Deruelle P, Debarge V, Ducloy-Bouthors A-S, Subtil D. Evaluation of a new technique of prophylactic cervical cerclage simplified from the Shirodkar cerclage: A pilot study. Journal of Gynecology Obstetrics and Human Reproduction. 2017;46(4):343-7.

42. Hume H, Rebarber A, Saltzman DH, Roman AS, Fox NS. Ultrasound-indicated cerclage: Shirodkar vs. McDonald. The Journal of Maternal-Fetal and Neonatal Medicine. 2012;25(12):2690-2.

43. Wong C-H, Chen C-P, Wang K-G, Sun F-J, Chen C-Y. Comparison of two cervical cerclages for the prevention of preterm birth and neonatal complications. The Journal of Maternal-Fetal and Neonatal Medicine. 2017;30(5):551-5. 
44. Otsuki K, Nakai A, Matsuda Y, Shinozuka N, Kawabata I, Makino Y, et al. Randomized trial of ultrasound-indicated cerclage in singleton women without lower genital tract inflammation. Journal of Obstetrics and Gynaecology Research. 2016;42(2):148-57.

45. Brown R, Gagnon R, Delisle M-F. No. 373-cervical insufficiency and cervical cerclage. Journal of Obstetrics and Gynaecology Canada. 2019;41(2):233-47.

46. Royal College of Obstetricians and Gynaecologists. Green-top Guideline: Cervical Cerclage 2011 Available from: https://www.rcog.org.uk/en/guidelines-research-services/guidelines/gtg60/.

\section{Hosted file}

Figure 1.pdf available at https://authorea.com/users/397137/articles/510138-mcdonald-versusshirodkar-cerclage-technique-in-the-prevention-of-preterm-birth-a-systematic-review-andmeta-analysis

\section{Hosted file}

Table 1.pdf available at https://authorea.com/users/397137/articles/510138-mcdonald-versusshirodkar-cerclage-technique-in-the-prevention-of-preterm-birth-a-systematic-review-andmeta-analysis

\section{Hosted file}

Table 2A and 2B.pdf available at https://authorea.com/users/397137/articles/510138-mcdonaldversus-shirodkar-cerclage-technique-in-the-prevention-of-preterm-birth-a-systematicreview-and-meta-analysis

\section{Hosted file}

Figure 2.pdf available at https://authorea.com/users/397137/articles/510138-mcdonald-versusshirodkar-cerclage-technique-in-the-prevention-of-preterm-birth-a-systematic-review-andmeta-analysis

\section{Hosted file}

Table 3.pdf available at https://authorea.com/users/397137/articles/510138-mcdonald-versusshirodkar-cerclage-technique-in-the-prevention-of-preterm-birth-a-systematic-review-andmeta-analysis 